\title{
Detection of CD133 expression in U87 glioblastoma cells using a novel anti-CD133 monoclonal antibody
}

\author{
DONGYANG WANG ${ }^{1}$, YUANXU GUO ${ }^{1}$, YANQING LI $^{1}$, WEILING LI ${ }^{1}$, \\ XIAOJING ZHENG ${ }^{1}$, HAIBIN XIA ${ }^{1}$ and QINWEN MAO ${ }^{2}$ \\ ${ }^{1}$ Co-Innovation Center for Qinba Regions' Sustainable Development, College of Life Sciences, \\ Shaanxi Normal University, Xi'an, Shaanxi 710062, P.R. China; ${ }^{2}$ Department of Pathology, \\ Northwestern University Feinberg School of Medicine, Chicago, IL 60611, USA
}

Received June 22, 2014; Accepted February 2, 2015

DOI: $10.3892 / \mathrm{ol} .2015 .3079$

\begin{abstract}
In glioblastomas, the surface glycoprotein CD133 (prominin-1) indicates the presence of cancer stem cells (CSCs), which are able to initiate tumor growth and are highly resistant to conventional chemo/radiotherapy. However, a number of studies have reported that certain CD133- glioma cells are able to self-renew and retain tumorigenic potential. In addition, the reliability of CD133 as a CSC marker is controversial due to inconsistent findings with regard to the prognostic values and distribution of CD133. Such controversies may be due to the detection limits using currently available anti-CD133 antibodies. In the present study, novel anti-human CD133 monoclonal antibodies (mAbs) were generated using two recombinant extracellular domains of human CD133: CD133 ectodomain 1 (amino acids 171-420) and CD133 ectodomain 2 (amino acids 507-716). One of the antibodies produced against CD133 ectodomain 2, C2E1, detected high expression levels of CD133 protein in glioblastoma U87 cells, in contrast to previous studies which did not detect CD133 expression in these cells. The cells exhibited a cytoplasmic distribution pattern of CD133 and produced a $95 \mathrm{kDa}$ band following western blot analysis. In addition, C2E1 was able to bind the full-length glycosylated CD133 on the cell surface and inhibit the proliferation of tumor cells. Therefore, this antibody may be a valuable tool to study CD133 as a CSC marker and may be significant in future cancer treatments.
\end{abstract}

Correspondence to: Professor Haibin Xia, Co-Innovation Center for Qinba Regions' Sustainable Development, College of Life Sciences, Shaanxi Normal University, 620 West Chang'an Avenue, Xi'an, Shaanxi 710062, P.R. China

E-mail: hbxia2001@163.com

Key words: CD133, cancer stem cell, monoclonal antibody, glioblastoma

\section{Introduction}

Glioblastomas (GBMs) are the most common and aggressive malignant primary brain tumors in humans. GBM accounts for $~ 51 \%$ of all primary gliomas (1), with an annual incidence of 2-3 cases per 100,000 individuals in Europe and North America (2). GBMs are usually diagnosed by magnetic resonance imaging, and confirmed by biopsy (3). Certain genetic signatures, including TP53, IDH1 or TPEN, are hypothesized to present potential biomarkers for GBM (4). At present, the standard treatment for newly diagnosed glioblastoma patients is surgical resection, followed by a combination of chemotherapy and radiotherapy $(5,6)$. The prognosis of patients with glioblastoma is poor, and the median survival time is 14.6 months (6). GBMs contain a subpopulation of cancer stem cells (CSCs) that are able to self-renew in vitro and initiate new tumors in vivo $(7,8)$. CSCs may also mediate radio- and chemo-resistance in $\operatorname{GBMs}(7,8)$.

Previous studies have hypothesized that the transmembrane glycoprotein, CD133 (also known as prominin-1), is a CSC marker in malignant brain tumors $(9,10)$. In addition, a number of studies have revealed that $\mathrm{CD}_{133^{+}}$cells, but not CD133- cells, exhibit stem cell-like and tumor-initiating properties $(9,10)$. In addition, a number of studies have shown that CD133 closely correlates with tumor size, a worse prognosis, higher rates of lymph node metastasis and resistance to adjuvant therapies (11-13). Therefore, decreasing the expression of CD133 or exposing the protein to certain antibodies, such as AC133, may inhibit tumor cell growth, cell motility, spheroid-forming capacity and tumorigenic ability $(14,15)$. However, other studies have obtained contradictory results (16-20). Further controversial results include inconsistent findings with regard to the prognostic value and distribution patterns of CD133 $(9,10,21-28)$. These controversies may be due to the detection limits of currently available anti-CD133 antibodies (20).

The aim of the present study was to advance understanding with regard to the significance of CD133 in GBM tumor biology. Thus, in the current study, novel anti-human CD133 monoclonal antibodies (mAbs) were generated using two recombinant extracellular domains of human CD133. In addition, the expression levels of CD133 protein in U87 
glioblastoma cells was detected using the produced antibodies.

\section{Materials and methods}

Cell culture and transfection. Human colonic carcinoma Caco-2 cells, human glioblastoma U87 cells and human embryonic kidney (HEK) 293 cells were obtained from the American Type Culture Collection (Manassas, VA, USA). All cells were cultured in Dulbecco's modified Eagle's medium (DMEM; Gibco Life Technologies, Grand Island, NY, USA) supplemented with $10 \%$ (vol/vol) fetal bovine serum (FBS; Gibco Life Technologies), $1 \%$ penicillin-streptomycin (MP Biomedicals, Santa Ana, CA, USA) and 1\% L-glutamate (MP Biomedicals). In addition, mouse myeloma cells, SP2/0 (American Type Culture Collection), were cultured in RPMI 1640 medium (Hyclone, Logan, UT, USA) supplemented with $10 \%$ FBS. The cell lines were maintained in a humidified atmosphere of $5 \% \mathrm{CO}_{2}$ at $37^{\circ} \mathrm{C}$. The standard calcium phosphate method (29) was used to transfect HEK 293 cells. The medium was replaced at $4 \mathrm{~h}$ post-transfection and the cells were analyzed at 24-48 h post-transfection.

Plasmid construction. The cDNA coding CD133 was isolated from the MegaMan Human Transcriptome Library (Agilent Technologies, Santa Clara, CA, USA) by polymerase chain reaction (PCR) using forward primer, 5'-aggatcc atggecetcgtactcggct-3', and reverse primer, 5'-tatcgatttaatgttgtgatgggettg-3'.

The amino acid sequences of CD133 ectodomain 1 (amino acids 171-420) and CD133 ectodomain 2 (amino acids 507-716) were selected from the ectodomains of CD133 based on its reported structure (Fig. 1A) (30). CD133 ectodomains 1 and 2 were amplified using the following primers: CD133 ectodomain 1 forward, 5'-ccatcgatatgagtcggaaactggcagatag-3', and reverse, 5'-gctctagattactgaataggaagacgctgag-3'; CD133 ectodomain 2 forward, 5'-ccatcgatatgtgtgaaccttacacgagca-3', and reverse, 5'-gactagtttagttctgagcaaaatccagag-3'.

PCR was performed in a $50 \mu 1$ reaction volume, consisting of $1 \mu \mathrm{l} \mathrm{cDNA}$ template, $10 \mathrm{mM}$ dNTPS (Takara Biotechnology Co., Ltd., Dalian, China), and 1 U LA Taq DNA polymerase (Takara Biotechnology Co., Ltd.) under the following conditions: 30 cycles of $94^{\circ} \mathrm{C}$ for $30 \mathrm{sec}, 98^{\circ} \mathrm{C}$ for $10 \mathrm{sec}, 55^{\circ} \mathrm{C}$ for $15 \mathrm{sec}$ and $72^{\circ} \mathrm{C}$ for $1 \mathrm{~min}$, followed by a $10 \mathrm{~min}$ extension step at $72^{\circ} \mathrm{C}$. All the PCR products were cloned into a pGEM-T easy vector (Promega Corporation, Madison, WI, USA). The positive clones were identified by double restriction enzyme digestion with BamHI/ClaI for full length CD133, ClaI/XbaI for CD133 ectodomain 1 and ClaI/SpeI for CD133 ectodomain 2, and sequencing was performed by the Beijing Genomics Institute (Shenzhen, China). All restriction enzymes were obtained from New England Biolabs (Ipswich, MA, USA). The DNA sequences of CD133 ectodomain 1 and CD133 ectodomain 2 were subsequently inserted into a prokaryotic expression vector, pRSET, including an N-terminal 6xHis-tag.

Establishment of a stable U87 cell line expressing full-length CD133. The CD133 full-length cDNA was inserted into a XhoI/NotI-digested, modified lentiviral vector with an mCherry reporter gene and a hygromycin resistance gene (designated as L-mCherry-Hygro-CD133; Addgene Inc., Cambridge, MA, USA). 293T cells were co-transfected, using the calcium phosphate method, with the L-mCherry-Hygro-CD133 plasmid, pVPack-vesicular stomatitis virus G (VSV-G) vector (Addgene, Cambridge, MA, USA) encoding the VSV-G surface gene, and the psPAX2 vector (Addgene), which provides the env and gag genes. At $4 \mathrm{~h}$ after transfection, the medium was replaced by fresh DMEM. Next, lentiviral supernatants were enriched to transduce the U87 cells in the presence of $10 \mu \mathrm{g} / \mathrm{ml}$ polybrene (Sigma-Aldrich, St. Louis, MO, USA). At $24 \mathrm{~h}$ after transfection, the transduced cells were selected using $750 \mu \mathrm{g} / \mathrm{ml}$ hygromycin and red fluorescence from the mCherry reporter gene. The resultant stable cell line was designated as U87-CD133 overexpression.

CD133 antigen generation. The pRSET vector expressing CD133 ectodomain 1 or 2 was transformed into BL21 (DE3) E. coli. The transformed bacteria were grown in lysogeny broth medium and were induced by $0.25 \mathrm{mM}$ isopropyl $\beta$-D-1-thiogalactopyranoside (MP Biomedicals) for $4 \mathrm{~h}$. Subsequently, the cells were precipitated, resuspended in cell lysis buffer $\left(50 \mathrm{mM} \mathrm{NaH}_{2} \mathrm{PO}_{4}, 10 \mathrm{mM}\right.$ Tris- $\mathrm{HCl}, 250 \mathrm{mM} \mathrm{NaCl}$, $5 \mathrm{mg} / \mathrm{ml}$ lysozyme; pH 8.0; Takara Biotechnology Co., Ltd.) and disrupted by two cycles of sonication on ice at $900 \mathrm{~W}$. The protein expression was analyzed by $12 \%$ SDS-PAGE using Coomasie blue (MP Biomedicals) staining and confirmed by western blot analysis. Recombinant proteins with the 6xHis-tag were purified using nickel-nitrilotriacetic acid beads.

Immunization and $m A b$ production. All the animal experiments used in the study were approved by the Institutional Animal Care and Use Committee of Shaanxi Normal University (Xi'an, China). In total, 20 female Balb/c mice (age, 6 weeks) were injected subcutaneously with recombinant protein CD133 ectodomain 1-6xHis or CD133 ectodomain 2-6xHis, which were emulsified in complete Freund's adjuvant (Sigma-Aldrich). Next, the mice were immunized with the same recombinant protein three times until a sufficient serum titer against CD133 was obtained, as analyzed by enzyme-linked immunosorbent assay (ELISA). Mice with a high serum titer were selected and boosted a fourth time prior to cell fusion.

The spleen cells of the immunized Balb/c mice were isolated and fused with SP2/0 myeloma cells using polyethylene glycol. Following fusion, the cells were cultured in hypoxanthine-aminopterin-thymidine (HAT) selection medium (Gibco Life Technologies). The HAT selection medium was refreshed every 2-3 days, and the selection medium was omitted after 6 days. Following a further 5 days, culture supernatants were screened for the production of antibodies against CD133 ectodomains 1 and 2 using ELISA.

Western blot analysis. Caco-2, U87 and U87-CD133 overexpression cells were lysed in radioimmunoprecipitation assay lysis buffer (Shaanxi Pioneer Biotech Co., Ltd., Xi'an, China). The samples were subjected to $12 \%$ SDS-PAGE and then blotted onto methanol-pretreated polyvinylidene difluoride membranes (EMD Millipore, Billerica, MA, USA). The membranes were incubated with mouse anti-human CD133 ectodomain 
A

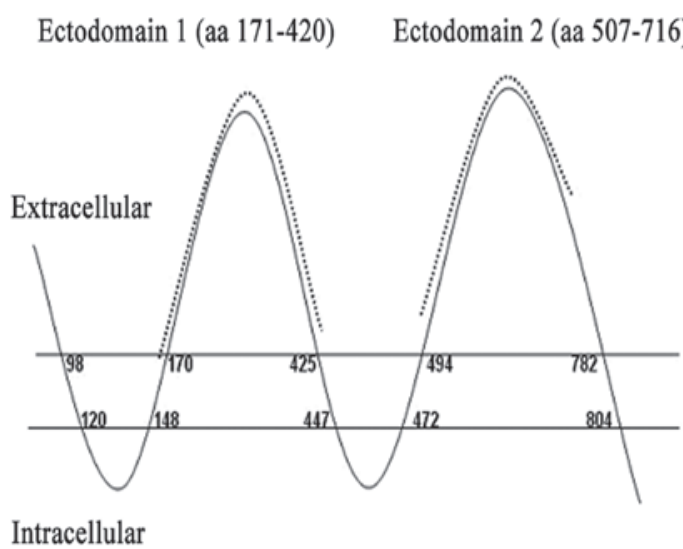

B

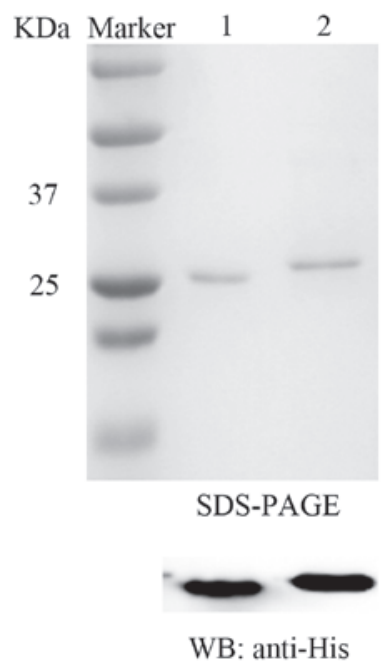

Figure 1. CD133 antigens used for mAb production. (A) Topological map of CD133 protein. Recombinant chimeric CD133 antigens, consisting of aa residues 171-420 and 507-716 (dotted line), were generated. (B) The two antigens, each tagged by an N-terminal 6xHis-tag, were expressed in E. coli and purified. The recombinant antigens were further verified by WB analysis with mouse anti-His mAb. Lane 1, ectodomain 1, Lane 2, ectodomain 2. CD133, prominin-1; mAb, monoclonal antibody; aa, amino acid; WB, Western blot.

mAbs (dilution, 1:300) for $90 \mathrm{~min}$ at room temperature. The rabbit anti-human CD133 mAb C24B9 (dilution, 1:1000; cat. no. 3663; Cell Signaling Technology, Danvers, MA, USA), which detects the full-length CD133 protein in Caco-2 cells (20), was used as a positive control. Next, the membranes were washed using phosphate-buffered saline (PBS)/Tween-20 (PBST) and incubated with horseradish peroxidase (HRP)-conjugated goat anti-mouse immunoglobulin $\mathrm{G}$ ( $\mathrm{IgG}$ ) polyclonal antibody (dilution, 1:10,000; cat. no. ZB-2305; Beijing Zhongshan Jinqiao Biological Technology Ltd., Beijing, China) or HRP-conjugated goat anti-rabbit IgG polyclonal antibody (dilution, 1:10,000; cat. no. ZB-2301; Beijing Zhongshan Jinqiao Biological Technology Ltd.). The membranes were further washed with PBST and visualized using the SuperSignal West Pico chemiluminescent substrate (Thermo Fisher Scientific, Hudson, NH, USA).

Fluorescent immunocytochemistry. HEK 293 cells transfected with CD133 full-length cDNA were fixed with $4 \%$ paraformaldehyde in PBS for $15 \mathrm{~min}$, followed by washing with PBS. The fixed cells were then incubated with mouse anti-human CD133 ectodomain mAbs (dilution, 1:50) for $2 \mathrm{~h}$ at room temperature. Following three washes with PBS, the cells were incubated with biotin-conjugated goat anti-mouse IgG polyclonal antibody (dilution, 1:100; cat. no. SA1072; Wuhan Boster Biological Technology, Ltd., Wuhan, China) for $30 \mathrm{~min}$ at $37^{\circ} \mathrm{C}$. The cells were further washed and incubated with streptavidin biotin complex (SABC)-Cy3 (dilution, 1:100; cat. no. SA1072; Wuhan Boster Biological Technology, Ltd.) for $15 \mathrm{~min}$ at $37^{\circ} \mathrm{C}$. Finally, the cells were briefly washed with PBS and visualized under a Zeiss Axio Observer Z1 inverted fluorescence microscope (Zeiss, Oberkochen, Germany).

Immunohistochemistry. U87 cells were fixed with 4\% paraformaldehyde in PBS for $15 \mathrm{~min}$. The cells were subsequently washed using PBS, blocked in normal horse serum (dilution, 1:200; cat. no. PK-6102; Vector Laboratories, Burlingame, CA, USA) and incubated with mouse anti-human CD133 ectodomain mAbs (dilution, 1:50) at $4^{\circ} \mathrm{C}$ overnight. After washing with PBS, the cells were incubated with biotin-conjugated horse anti-mouse IgG polyclonal antibody (dilution, 1:100; cat. no. PK-6102; Vector Laboratories) for $30 \mathrm{~min}$ at $37^{\circ} \mathrm{C}$. The cells were rinsed with PBS prior to incubation with HRP-conjugated SABC (dilution, 1:100; cat. no. PK-6102; Vector Laboratories) for $30 \mathrm{~min}$ at $37^{\circ} \mathrm{C}$. The 3,3'-diaminobenzidine chromogen (cat. no. ZLI-9017; Beijing Zhongshan Jinqiao Biological Technology Ltd.) was used for visualization under an inverted fluorescence microscope (DMIL LED; Leica, Wetzlar, Germany).

Cell proliferation assay. An MTT assay was performed according to a previously reported procedure (31), with minor modifications. Briefly, cells in $100 \mu \mathrm{l}$ medium were seeded in 96-well flat-bottom plates and supplemented with $50 \mu \mathrm{lmAbs}$ at various dilutions. Following a three-day incubation period, $15 \mu \mathrm{l}$ MTT solution (5 mg MTT/ml of distilled water) were added to each well, followed by incubation for a further $4 \mathrm{~h}$. Following removal of the supernatant, $150 \mu$ l dimethyl sulfoxide was used to dissolve the MTT crystals in the wells. Finally, the plates were subjected to cell viability detection using an ELISA reader (Multiskan EX; Thermo Fisher Scientific) at a wavelength of $570 \mathrm{~nm}$. Optical density (OD) at $570 \mathrm{~nm}$ [absorbance value at 570nm (OD570)] was examined to analyze cell proliferation.

Statistical analysis. All data are presented as the mean + standard deviation (SD) from at least three different experiments. One way analysis of variance was used to investigate differences between the experimental and control groups. All statistical analyses were performed using SPSS 13.0 software (SPSS, Inc., Chicago, IL, USA).

\section{Results}

Generation and purification of CD133 antigens. CD133 amino acid fragments 171-420 (CD133 ectodomain 1) and 507-716 (CD133 ectodomain 2) were selected as the CD133 
A
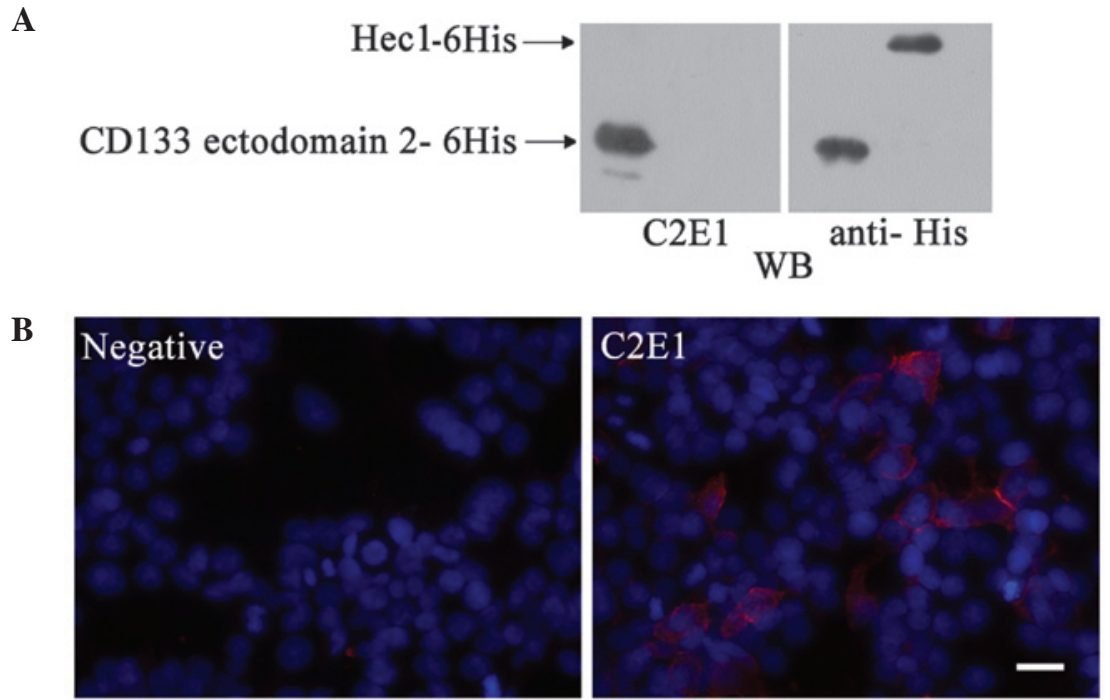

Figure 2. Validation of CD133 hybridoma cell lines. (A) The purified CD133 ectodomain 2-His and control Hec1-6xHis fusion protein were blotted from the SDS-PAGE gel onto a polyvinylidene difluoride and probed with C2E1 and control anti-His monoclonal antibodies. (B) Immunofluorescence staining with C2E1 of HEK 293 cells transfected with a plasmid expressing CD133. Pre-immunized serum served as a negative control. Scale bar, $20 \mu \mathrm{m}$. CD133, prominin-1; WB, western blot.

$\mathbf{A}$

$115 \mathrm{KDa}$

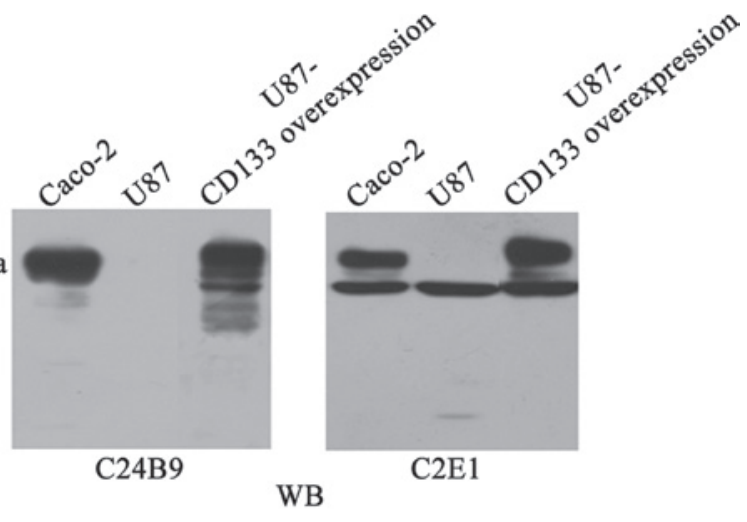

B

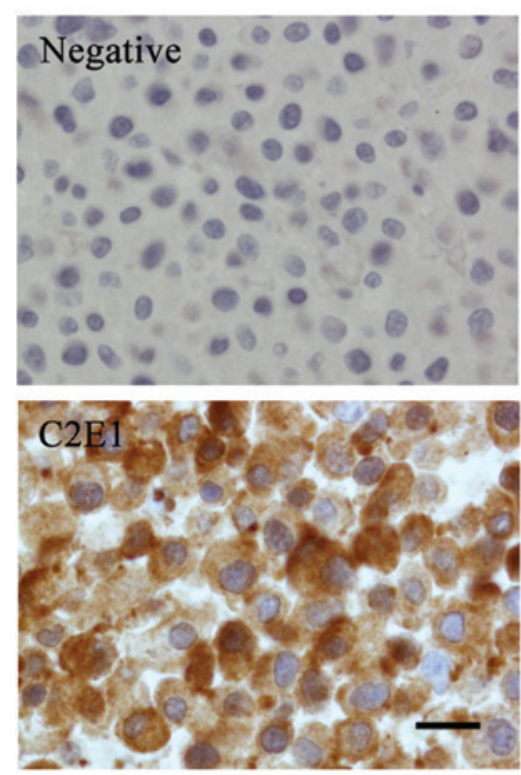

Figure 3. CD133 expression in glioblastoma U87 cells. (A) The cell lysates of U87, U87-CD133 overexpression and the control cell line Caco-2 were analyzed by WB analysis using C2E1 and the commercially-available antibody, C24B9. (B) Immunohistochemical staining with C2E1 revealed endogenous $\mathrm{CD} 133$ expression in the U87 cells. Pre-immunized serum served as a negative control. Scale bar, $20 \mu \mathrm{m}$. CD133, prominin-1; WB, western blot.
A

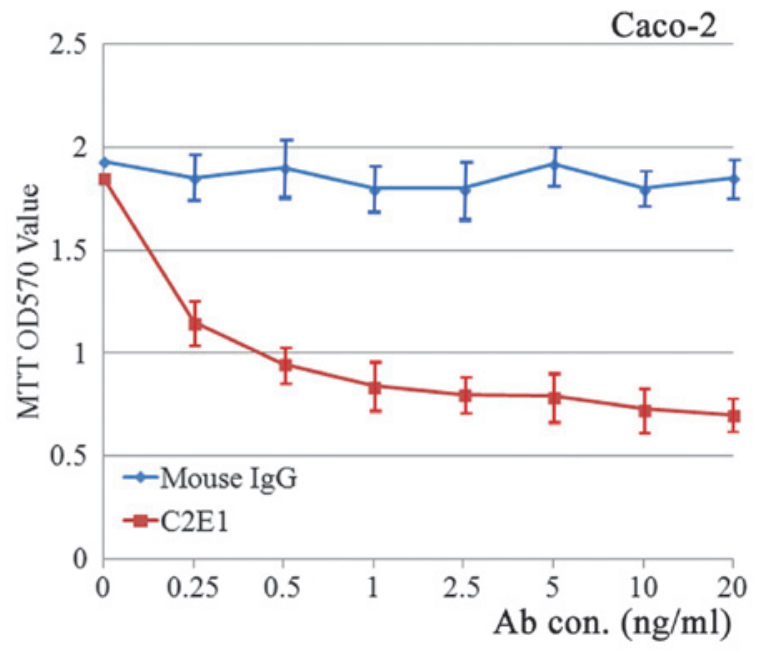

B

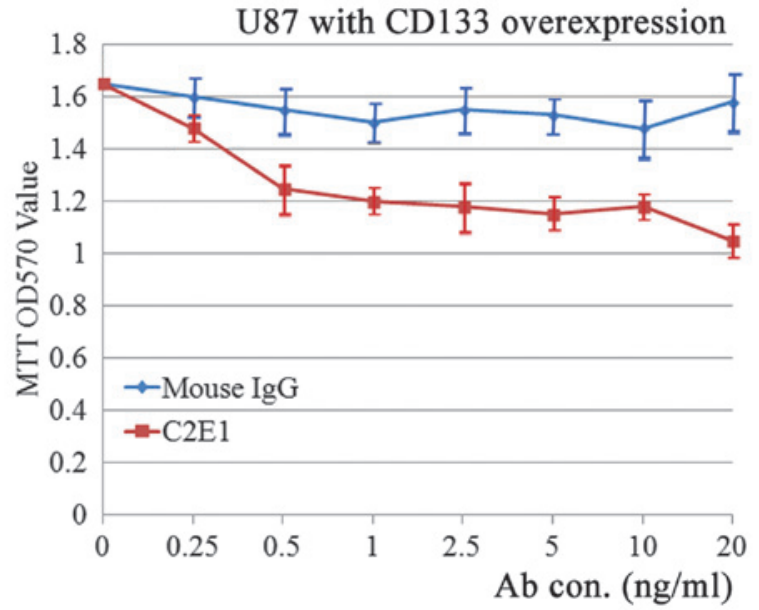

Figure 4. The effect of C2E1 on the proliferation of (A) Caco-2 and (B) U87-CD133 overexpression cells. Proliferation curves were determined using an MTT assay. Values are expressed as the mean \pm standard error of four treatments. The highest inhibitory rates were $63.3 \pm 2.93 \%(\mathrm{P}<0.01)$ for the Caco- 2 cells and $33.3 \pm 4.73 \%(\mathrm{P}<0.05)$ for the U87-CD133 overexpression cells. CD133, prominin-1; Ab con, antibody concentration; IgG, immunoglobulin G. 
antigens. The locations of these two amino acid fragments in the CD133 protein are illustrated in Fig. 1A. The two CD133 ectodomain antigens, which were tagged by an N-terminal 6xHis-tag, were expressed in E. coli, purified and analyzed by SDS-PAGE (Fig. 1B). The purified recombinant proteins were further verified using western blot analysis with mouse anti-His mAb (Fig. 1B).

Generation and validation of CD133 mAbs. The purified antigens were injected into Balb/c mice to generate monoclonal anti-CD133 antibodies using standard hybridoma technology. ELISA was used to screen cell culture supernatants from the resulting hybridoma clones for the production of antibodies against recombinant CD133. At least six positive stable clones secreting anti-CD133 antibodies were obtained (data not shown). The positive hybridoma clones were validated by western blot analysis using prokaryotically-expressed CD133 ectodomain 1 and CD133 ectodomain 2 recombinant proteins. Four hybridoma clones against CD133 ectodomain 1 and two clones against ectodomain 2 were identified. HEK 293 cells were transfected with an expression plasmid encoding the human CD133 full-length cDNA and stained immunohistochemically with different hybridoma clones. One of the hybridoma clones, $\mathrm{C} 2 \mathrm{E} 1$, which recognized the recombinant CD133 ectodomain 2 specifically, exhibited cell membrane and cytoplasmic staining in the transfected HEK 293 cells (Fig. 2).

Novel anti-CD133 mAb detects CD133 expression in the glioblastoma U87 cell line. CD133 expression in U87 cells was screened with each of the obtained anti-CD133 mAbs. The $\mathrm{CD}_{133^{+}}$colorectal adenocarcinoma cell line, Caco-2, was used as a positive control and the commercially available anti-CD133 mAb, C24B9, was used as a positive control. Similar to C24B9, all the hybridoma clones detected a $115 \mathrm{kDa}$ glycosylated full-length CD133 protein in Caco-2 cells, but were unable to detect a considerable CD133 expression in U87 cells, with the exception of C2E1 (Fig. 3A). In the C2E1 clones, a $95 \mathrm{kDa}$ band was revealed in Caco-2 and U87 cells, in addition to the $115 \mathrm{kDa}$ band in Caco-2 cells (Fig. 3A). A stable U87 cell line expressing full-length CD133 (termed as U87-CD133 overexpression) was established by infecting native U87 cells with CD133-expressing lentivirus, followed by selection. Western blot analysis with C2E1 and C24B9 demonstrated glycosylated full-length CD133 bands in the U87-CD133 overexpression cell line (Fig. 3A). Cellular distribution of endogenous CD133 expression in the native U87 cells was evaluated by immunohistochemical staining with $\mathrm{C} 2 \mathrm{E} 1$, which revealed a cytoplasmic distribution pattern (Fig. 3B).

In vitro biological effects of the novel anti-CD133 $\mathrm{mAb}$ on cancer cells. To investigate whether the C2E1 antibody exhibits a biological activity (for instance, neutralization of the membranous CD133 protein), the Caco-2, native U87 and U87-CD133 overexpression cells were incubated with increasing concentrations of C2E1 for $72 \mathrm{~h}$. An MTT assay revealed that incubation with $\mathrm{C} 2 \mathrm{E} 1$ inhibited the proliferation of Caco-2 cells in a dose-dependent manner (Fig. 4A). The inhibitory rate was calculated using the following formula: Inhibitory rate $(\%)=($ OD570 value of normal mouse IgG - OD570 value of C2E1)/(OD570 value of normal mouse $\operatorname{IgG}) \times 100$. The highest inhibitory rate was $63.3 \pm 2.93 \%(\mathrm{P}<0.01)$, which was observed when the antibody concentration reached $20 \mathrm{ng} / \mathrm{ml}$. However, C2E1 had no effect on the proliferation of U87 cells (data not shown), which may be due to the predominantly cytoplasmic distribution of CD133 protein in native U87 cells. As predicted, U87-CD133 overexpression cells responded to the cell growth inhibitory effect of C2E1 (Fig. 4B). The highest inhibitory rate was $33.3 \pm 4.73 \%(\mathrm{P}<0.05)$, which was observed when the antibody concentration reached $20 \mathrm{ng} / \mathrm{ml}$. Notably, the cell proliferation rate of untreated U87-CD133 overexpression cells was similar to that of native U87 cells.

\section{Discussion}

U87, a commonly-used commercial cell line for glioblastoma research, exhibits the behaviors and features of CSCs $(32,33)$. However, previous studies have reported that the U87 cell line does not express the CSC marker, CD133, upon employing standard anti-CD133 antibodies, including AC133, 293 C3 (34) or W6B3C1 (35). To the best of our knowledge, in the current study, an anti-human CD133 mAb was constructed that detected a $95 \mathrm{kDa}$ endogenous CD133 protein with a cytoplasmic distribution pattern in U87 cells for the first time.

A number of studies have reported inconsistent immunolabeling of CSCs upon using different CD133 antibodies. This inconsistency may be associated with the glycosylation status and variants of the CD133 glycoprotein $(36,37)$. Tumor stem cells may change the glycosylation status of CD133 upon differentiation $(28,36,38)$. Antibodies that recognize the glycosylated epitopes may therefore detect only a limited subset of $\mathrm{CD} 133^{+}$cells. In addition, $\geq 28$ alternatively spliced CD133 variants have been identified (39-41). As different CD133 antibodies do not recognize all the splice variants, this may explain the inconsistencies in immunolabeling and varying protein sizes in different studies (20).

In the present study, the novel $\mathrm{mAb} \mathrm{C} 2 \mathrm{E} 1$ recognized a CD133 protein product of U87 cells with a molecular weight of $95 \mathrm{kDa}$, which has a similar size to the non-glycosylated full-length CD133. However, the $95 \mathrm{kDa}$ CD133 in U87 cells may be a CD133 variant rather than the non-glycosylated full-length CD133, since the latter should be detected by the 'pan'-C24B9 antibody (20). Furthermore, the cytoplasmic distribution of CD133 in U87 cells revealed by C2E1 immunostaining indicated that this protein is not likely to be glycosylated.

Whether this novel CD133 antigen plays a role in the stem cell behavior of U87 cells, or whether it is present in other CSCs, remains unclear. CD133 analysis must not focus on specific glycosylated epitopes, but instead cover all the CD133 variants. The inclusion of different CD133 variants may produce more consistent results in the study of the CD133 biology in CSCs (20). C2E1, the novel anti-CD133 mAb that was produced in the current study, is able to recognize the $95 \mathrm{kDa}$ CD133 variant and also bind full-length glycosylated CD133. Therefore, this novel mAb may be a valuable tool for the study of CD133 as a CSC marker.

An important advance in cancer therapy is the development of targeted agents that are able to neutralize the CSC response to circulating growth factors. The results of the current study indicated that incubation with $\mathrm{C} 2 \mathrm{E} 1$ may inhibit proliferation in Caco-2 and U87-CD133 overexpression cells, indicating 
that $\mathrm{C} 2 \mathrm{E} 1$ exerts a neutralizing effect on $\mathrm{CD} 133^{+}$cells; this may be of significance in the development of cancer therapies.

In conclusion, the novel anti-CD133 mAb, C2E1, indicated that endogenous CD133 expression is present in glioblastoma U87 cells. This mAb is able to bind cell surface CD133 and inhibit the proliferation of tumor cells.

\section{Acknowledgements}

This study was supported by the Fundamental Research Funds for the Central Universities (grant nos. GK201301010 and GK201104004), the Innovation Funds of Graduate Programs, Shaanxi Normal University (grant no. 2011CXB006) and research grants to Haibin Xia and Xiaojing Zheng from the National Natural Science Foundation of China (grant nos. 81272543, 81471772 and 81301957).

\section{References}

1. Ostrom QT, Gittleman H, Liao P, et al: CBTRUS statistical report: primary brain and central nervous system tumors diagnosed in the United States in 2007-2011. Neuro Oncol 16 (Suppl 4): iv1-iv63, 2014.

2. Verdecchia A, De Angelis G and Capocaccia R: Estimation and projections of cancer prevalence from cancer registry data. Stat Med 21: 3511-3526, 2002.

3. Weller M: Novel diagnostic and therapeutic approaches to malignant glioma. Swiss Med Wkly 141: w13210, 2011.

4. McNamara MG, Sahebjam S and Mason WP: Emerging biomarkers in glioblastoma. Cancers (Basel) 5: 1103-1119, 2013.

5. Stupp R, Hegi ME, Mason WP, et al; European Organisation for Research and Treatment of Cancer Brain Tumour and Radiation Oncology Groups; National Cancer Institute of Canada Clinical Trials Group: Effects of radiotherapy with concomitant and adjuvant temozolomide versus radiotherapy alone on survival in glioblastoma in a randomised phase III study: 5-year analysis of the EORTC-NCIC trial. Lancet Oncol 10: 459-466, 2009.

6. Stupp R, Mason WP, van den Bent MJ, et al; European Organisation for Research and Treatment of Cancer Brain Tumor and Radiotherapy Groups; National Cancer Institute of Canada Clinical Trials Group: Radiotherapy plus concomitant and adjuvant temozolomide for glioblastoma. N Engl J Med 352: 987-996, 2005.

7. Jordan CT, Guzman ML and Noble M: Cancer stem cells. N Engl J Med 355: 1253-1261, 2006.

8. Reya T, Morrison SJ, Clarke MF and Weissman IL: Stem cells, cancer, and cancer stem cells. Nature 414: 105-111, 2001.

9. Singh SK, Hawkins C, Clarke ID, et al: Identification of human brain tumour initiating cells. Nature 432: 396-401, 2004.

10. Singh SK, Clarke ID, Terasaki M, et al: Identification of a cancer stem cell in human brain tumors. Cancer Res 63: 5821-5828, 2003.

11. Lim SH, Jang J, Park JO, et al: CD133-positive tumor cell content is a predictor of early recurrence in colorectal cancer. $\mathrm{J}$ Gastrointest Oncol 5: 447-456, 2014.

12. Mizugaki H, Sakakibara-Konishi J, Kikuchi J, et al: CD133 expression: a potential prognostic marker for non-small cell lung cancers. Int J Clin Oncol 19: 254-259, 2014.

13. Hashimoto K, Aoyagi K, Isobe T, et al: Expression of CD133 in the cytoplasm is associated with cancer progression and poor prognosis in gastric cancer. Gastric Cancer 17: 97-106, 2014.

14. Rappa G, Fodstad $\mathrm{O}$ and Lorico A: The stem cell-associated antigen CD133 (Prominin-1) is a molecular therapeutic target for metastatic melanoma. Stem Cells 26: 3008-3017, 2008.

15. Wang CH, Chiou SH, Chou CP, et al: Photothermolysis of glioblastoma stem-like cells targeted by carbon nanotubes conjugated with CD133 monoclonal antibody. Nanomedicine 7: 69-79, 2011.

16. Clément V, Marino D, Cudalbu C, et al: Marker-independent identification of glioma-initiating cells. Nat Methods 7: 224-228, 2010.

17. Clément V, Dutoit V, Marino D, Dietrich PY and Radovanovic I: Limits of CD133 as a marker of glioma self-renewing cells. Int J Cancer 125: 244-248, 2009.

18. Wang J, Sakariassen $P \emptyset$, Tsinkalovsky O, et al: CD133 negative glioma cells form tumors in nude rats and give rise to CD133 positive cells. Int J Cancer 122: 761-768, 2008.
19. Beier D, Hau P, Proescholdt M, et al: CD133(+) and CD133(-) glioblastoma-derived cancer stem cells show differential growth characteristics and molecular profiles. Cancer Res 67: 4010-4015, 2007

20. Hermansen SK, Christensen KG, Jensen SS and Kristensen BW: Inconsistent immunohistochemical expression patterns of four different CD133 antibody clones in glioblastoma. J Histochem Cytochem 59: 391-407, 2011.

21. Zeppernick F, Ahmadi R, Campos B, et al: Stem cell marker CD133 affects clinical outcome in glioma patients. Clin Cancer Res 14: 123-129, 2008.

22. Immervoll H, Hoem D, Sakariassen PØ, Steffensen OJ and Molven A: Expression of the 'stem cell marker' CD133 in pancreas and pancreatic ductal adenocarcinomas. BMC Cancer 8: 48, 2008.

23. Thon N, Damianoff K, Hegermann J, et al: Presence of pluripotent $\mathrm{CD}_{133^{+}}$cells correlates with malignancy of gliomas. Mol Cell Neurosci 43: 51-59, 2010.

24. Beier D, Wischhusen J, Dietmaier W, et al: CD133 expression and cancer stem cells predict prognosis in high-grade oligodendroglial tumors. Brain Pathol. 18: 370-377, 2008.

25. Pallini R, Ricci-Vitiani L, Banna GL, et al: Cancer stem cell analysis and clinical outcome in patients with glioblastoma multiforme. Clin Cancer Res 14: 8205-8212, 2008.

26. Christensen K, Schrøder HD and Kristensen BW: CD133 identifies perivascular niches in grade II-IV astrocytomas. J Neurooncol 90: 157-170, 2008.

27. Bidlingmaier S, Zhu X and Liu B: The utility and limitations of glycosylated human CD133 epitopes in defining cancer stem cells. J Mol Med (Berl) 86: 1025-1032, 2008.

28. Florek M, Haase M, Marzesco AM, et al: Prominin-1/CD133, a neural and hematopoietic stem cell marker, is expressed in adult human differentiated cells and certain types of kidney cancer. Cell Tissue Res 319: 15-26, 2005.

29. Jordan M, Schallhorn A and Wurm FM: Transfecting mammalian cells: optimization of critical parameters affecting calcium-phosphate precipitate formation. Nucleic Acids Res 24: 596-601, 1996.

30. Shmelkov SV, St Clair R, Lyden D and Rafii S: AC133/CD133/Prominin-1. Int J Biochem Cell Biol 37: 715-719, 2005.

31. Plumb JA, Milroy R and Kaye SB: Effects of the $\mathrm{pH}$ dependence of 3-(4,5-dimethylthiazol-2-yl)-2,5-diphenyl-tetrazolium bromide-formazan absorption on chemosensitivity determined by a novel tetrazolium-based assay. Cancer Res 49: 4435-4440, 1989.

32. Campos B and Herold-Mende CC: Insight into the complex regulation of CD133 in glioma. Int J Cancer 128: 501-510, 2011.

33. Wu A, Oh S, Wiesner SM, et al: Persistence of CD133+ cells in human and mouse glioma cell lines: detailed characterization of GL261 glioma cells with cancer stem cell-like properties. Stem Cells Dev 17: 173-184, 2008.

34. Platet N, Liu SY, Atifi ME, et al: Influence of oxygen tension on CD133 phenotype in human glioma cell cultures. Cancer Lett 258: 286-290, 2007.

35. Christensen K, Aaberg-Jessen C, Andersen C, Goplen D, Bjerkvig $\mathrm{R}$ and Kristensen BW: Immunohistochemical expression of stem cell, endothelial cell, and chemosensitivity markers in primary glioma spheroids cultured in serum-containing and serum-free medium. Neurosurgery 66: 933-947, 2010.

36. Corbeil D, Röper K, Hellwig A, et al: The human AC133 hematopoietic stem cell antigen is also expressed in epithelial cells and targeted to plasma membrane protrusions. J Biol Chem 275: 5512-5520, 2000.

37. Miraglia S, Godfrey W, Yin AH, et al: A novel five-transmembrane hematopoietic stem cell antigen: isolation, characterization, and molecular cloning. Blood 90: 5013-5021, 1997.

38. Mizrak D, Brittan M and Alison M: CD133: molecule of the moment. J Pathol 214: 3-9, 2008.

39. Fargeas CA, Huttner WB and Corbeil D: Nomenclature of prominin-1 (CD133) splice variants - an update. Tissue Antigens 69: 602-606, 2007.

40. Jaszai J, Fargeas CA, Florek M, Huttner WB and Corbeil D: Focus on molecules: prominin-1 (CD133). Exp Eye Res 85: 585-586, 2007.

41. Fargeas CA, Joester A, Missol-Kolka E, Hellwig A, Huttner WB and Corbeil D: Identification of novel Prominin-1/CD133 splice variants with alternative C-termini and their expression in epididymis and testis. J Cell Sci 117: 4301-4311, 2004. 